\title{
PG0946 + 301 - A low-redshift, broad absorption line QSO
}

\section{Citation}

Wilkes, B. J. 1985. “PG0946 + 301 - A Low-Redshift, Broad Absorption Line QSO." The Astrophysical Journal 288 (January): L1. doi:10.1086/184409.

\section{Published Version}

10.1086/184409

\section{Permanent link}

http://nrs.harvard.edu/urn-3:HUL.InstRepos:32955253

\section{Terms of Use}

This article was downloaded from Harvard University's DASH repository, and is made available under the terms and conditions applicable to Other Posted Material, as set forth at http:// nrs.harvard.edu/urn-3:HUL.InstRepos:dash.current.terms-of-use\#LAA

\section{Share Your Story}

The Harvard community has made this article openly available.

Please share how this access benefits you. Submit a story.

Accessibility 


\title{
PG 0946 + 301: A LOW-REDSHIFT, BROAD ABSORPTION LINE QSO
}

\author{
BELINDA J. WILKES ${ }^{1}$ \\ Harvard-Smithsonian Center for Astrophysics \\ Received 1984 August 29; accepted 1984 October I
}

\begin{abstract}
The discovery of a new broad absorption line (BAL) QSO with the lowest emission-line redshift to date $(z=1.216)$ is reported. The data provide evidence for complex broad absorption up to velocities $\sim 14,500$ $\mathrm{km} \mathrm{s}^{-1}$ relative to the emission-line center.
\end{abstract}

Subject heading: quasars

\section{INTRODUCTION}

The two original members of the class of broad absorption line (BAL) QSOs, PHL 5200 (Lynds 1967) and RS 23 (Burbidge 1970), were discovered in the early days of QSO history. Since no more were discovered for many years, they were thought to be a rare or peculiar variety of QSO. The emission and absorption profiles in both these objects are similar to the P Cygni profiles from expanding envelopes of stars, and interpretation along these lines was invoked. More recently, increasing numbers of BAL QSOs have been discovered, mainly as a result of the development of objective prism survey techniques. The number of members in the class has doubled in the past 2 years: the review by Weymann, Carswell, and Smith (1981) lists 18 objects; that of Weymann and Foltz (1983) refers to 33 known BALs; and the number is still increasing.

The majority of the broad absorption lines originate in highly ionized gas, the most prominent lines being $O$ VI $\lambda 1034$, $\mathrm{N}$ v $\lambda 1240, \mathrm{Si}$ IV $\lambda 1397$, and C IV $\lambda 1549$. These are all ultraviolet lines with no counterparts at visual wavelengths; consequently, all BAL QSOs to date are at high redshift $z \geq 1.6$ (such that these lines are shifted to visual wavelengths) and are relatively faint $\left(m_{v}>17\right)$. In a few cases, lower ionization lines are also visible in absorption, e.g., Al III $\lambda 1858$ and Mg II $\lambda 2798$ (Foltz et al. 1983 b); generally their velocity width is significantly smaller than that of the high-ionization lines. Searches for lower redshift objects using IUE have so far proved fruitless.

The QSO, PG 0946+301, was discovered by Schmidt and Green (1983), who report a redshift of 1.216. In making more detailed observations, with the aim of studying the emissionline profiles, the observed wavelength range was extended down to the atmospheric cutoff $(\sim 3100 \AA)$, uncovering broad absorption shortward of the $C$ IV emission line.

\section{OBSERVATIONS}

The spectroscopic data presented in this paper were obtained in 1984 March on the UAO 90 inch $(2.28 \mathrm{~m})$ telescope using a dual-beam, intensified, photon-counting Reticon de-

\footnotetext{
${ }^{1}$ Visiting Scientist at Steward Observatory, University of Arizona.
}

tector mounted on the back of the Boller \& Chivens spectrograph. The spectrograph entrance apertures were circular holes with a projected diameter of 2 ".5. An 832 grooves per millimeter grating was used, yielding a well-calibrated wavelength range of $3150-4300 \AA$ at a resolution of $\sim 1.5 \AA$. The data were reduced to a linear wavelength scale in the usual manner, and a rough flux calibration performed by comparison with observations of the star EG 63 (Oke 1974) earlier on the same night. It should be noted that no attempt was made to determine absolute spectrophotometry since the entrance apertures were of comparable size to the seeing disk, and thus an indeterminate amount of light was lost. The total exposure time is $3200 \mathrm{~s}$, and the data are presented in photon flux units (i.e., photons $\mathrm{cm}^{-2} \mathrm{~s}^{-1} \AA^{-1}$ ) in Figure 1 .

\section{RESULTS}

This QSO was discovered as part of the Palomar Bright QSO Survey (BQS) reported by Schmidt and Green (1983); accurate coordinates and finding charts may be found therein. They determine a redshift of 1.216 based upon the $\mathrm{C}$ III] $\lambda 1909$ and $\mathrm{Mg}$ II $\lambda 2798$ emission lines in a low-resolution spectrum covering the wavelength range $3600-6800 \AA$. The high-resolution $(\sim 1.5 \AA)$ spectrum reported here extends from the atmospheric cutoff, $\sim 3150 \AA$ in the UV to $4300 \AA$, just covering the $C$ III] emission line and allowing detection of the C IV emission line and the broad absorption trough shortward of this emission. With a redshift of 1.216, this is the lowest redshift BAL QSO yet known. Its apparent magnitude ( $B=16.0$ ) also puts it among the most luminous of the BALs.

The spectrum (Fig. 1) shows the $C$ IV absorption trough having a sharp, low-velocity edge $\left(\Delta v \approx 1600 \mathrm{~km} \mathrm{~s}^{-1}\right)$ which substantially contaminates the emission-line profile being only $\sim 2500 \mathrm{~km} \mathrm{~s}^{-1}$ from the line peak (see Table 1). The trough is flat bottomed with a considerable amount of structure. The high-velocity edge is more gradual than the low $(\Delta v \sim 3700$ $\mathrm{km} \mathrm{s}^{-1}$ ) and has a possible subsidiary trough (labeled $\mathrm{C}$ in Fig. 1) embedded in it. The full velocity width of absorption trough is $\sim 12,100 \mathrm{~km} \mathrm{~s}^{-1}$, the high-velocity edge being $\sim 14,500 \mathrm{~km} \mathrm{~s}^{-1}(0.05 c)$ from the emission-line center. From these characteristics, there is no doubt that the object qualifies as a BAL QSO under the criteria proposed by Weymann, 


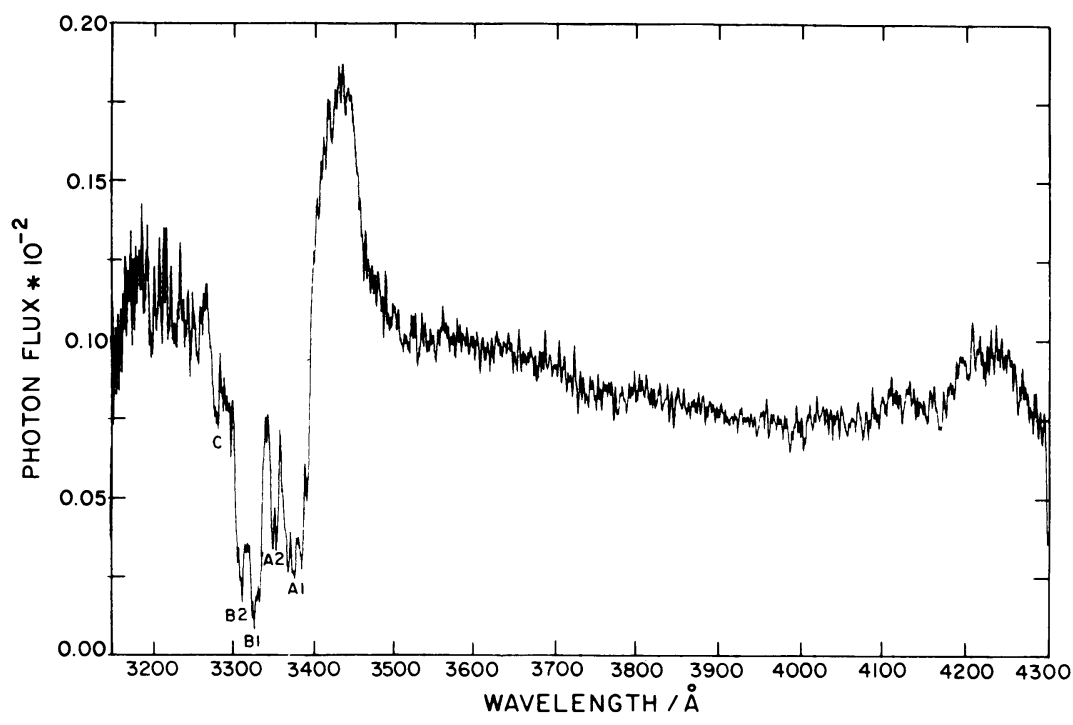

FIG. 1. - Spectrum of PG $0946+301$ obtained in 1984 March on the UAO $90^{\prime \prime}$ telescope

Carswell, and Smith (1981) that absorption should be spread over $>2000 \mathrm{~km} \mathrm{~s}^{-1}$ and with a maximum velocity $>5000$ $\mathrm{km} \mathrm{s}^{-1}$ relative to the emission-line peak. It is best classified as a class IV type following the scheme outlined by Weymann and Foltz (1983), which is based upon the degree of structure of the absorption.

The main absorption trough is most simply characterized as two clumps of absorbing systems each having two subsidiary components. These have been labeled A1, A2, B1, and B2 in Figure 1. The estimated central position of each of the five subsidiary troughs (including $\mathrm{C}$ mentioned above) is tabulated in Table 1 along with its velocity with respect to the emissionline redshift as measured from this spectrum (1.214) (see Table 2). Also listed are the estimated position and velocity of the low- and high-velocity extremities of the full trough. Al III $\lambda 1858$ absorption, corresponding to part or all of the C IV absorption, has been observed in a few BAL QSOs (e.g., $1011+091$; Foltz et al. 1983b); none is present for this object. Schmidt and Green's (1983) spectrum of PG $0946+301$ included a poor quality scan of the $\mathrm{Mg}$ II emission line, and no broad absorption was detected (Green 1984). Further observations are underway to obtain improved limits on the presence of broad $\mathrm{Mg}$ II absorption, since, when seen, it is generally weaker and narrower than that of the high-ionization lines. But at present there is no corresponding absorption in another species with which to compare the structure of the C IV absorption.

It has been noted that the $\mathrm{C}$ III] emission profile tends to be broader in BAL QSOs than in non-BAL QSOs (Turnshek 1984) and also that the emission lines tend to have a weaker peak intensity relative to the continuum when there is structure in the absorption trough (Weymann and Foltz 1983). There is little quantitative evidence for this trend available since the emission profiles are frequently contaminated by absorption, rendering measurement impossible. Qualitatively, this QSO follows the general trend; useful measurements are here precluded by the absorption contamination of $\mathrm{C}$ IV and the lack of a longward continuum for $\mathrm{C}$ III]. One possible
TABLE 1

Position of the Components of the Broad Absorption Troughs AND THEIR VELOCITY WITH RESPECT TO THE MEAN EMISSION LINE REDSHIFT (1.214)

\begin{tabular}{|c|c|c|}
\hline ID & $\lambda / \AA( \pm 0.5)$ & $\Delta v / \mathrm{km} \mathrm{s}^{-1}( \pm 50)$ \\
\hline Low-velocity edge ........ & 3402.5 & 2400 \\
\hline Al $\ldots \ldots \ldots \ldots \ldots$ & 3372.5 & 5040 \\
\hline $\mathrm{A} 2 \ldots \ldots \ldots$ & 3350.0 & 7060 \\
\hline B1. & 3325.0 & 9300 \\
\hline B2 & 3310.0 & 10650 \\
\hline C.. & 3277.5 & 13600 \\
\hline High-velocity edge .... & 3267.5 & 14500 \\
\hline
\end{tabular}

TABLE 2

Position and Redshift of the Emission Features

\begin{tabular}{|c|c|c|}
\hline Line & $\lambda / \AA ̊ \AA( \pm 0.5)$ & $z$ \\
\hline C IV $\lambda 1549 \ldots$ & 3430.0 & 1.214 \\
\hline $\mathrm{Al} \mathrm{III]}] \lambda 1858 \ldots \ldots$ & 4120.0 & 1.217 \\
\hline$C_{\text {III }} \lambda 1909 \ldots \ldots \ldots$ & 4225.0 & 1.213 \\
\hline
\end{tabular}

contributor to the larger width of the $\mathrm{C}$ III] profile is contamination by $\mathrm{Al}$ III $\lambda 1858$ emission in its shortward wing. This line has occasionally been observed in non-BAL QSOs (Wilkes 1984) but appears to be more common in the BAL QSOs (e.g., Foltz et al. 1983a). An emission feature is present in Figure 1 at $\sim 4120 \AA$ which is probably due to $\mathrm{Al}$ III. The positions of the emission lines are listed in Table 2; the adopted mean emission redshift of 1.214 was computed from the present data without using the $\mathrm{Al}$ III position since its identification is not definitive.

A small number of BAL QSOs have been reported to have higher than average optical polarization [e.g., PHL 5200 $\left(\lambda 5000_{\mathrm{obs}}\right) \approx 6.2 \%$; cf. typical $\mathrm{QSO} \approx 0.5 \%$ (Stockman, Angel, and Hier 1981)]. The broad-band polarization of PG $0946+301$ has been observed to be $0.79 \pm 0.19 \%$ (Schmidt 1984), which is consistent with the observations of typical 
QSOs. This object would not be distinguishable from normal QSOs due to its polarization alone.

\section{CONCLUSIONS}

Although there are many strong selection effects biased toward finding BAL QSOs at $z>1.6$, the lack of any member of the class at lower redshifts has been suggestive that they do not exist at $z<1.6$. The observations reported here show that BAL QSOs do exist down to the lowest redshift at which they can be observed in the optical. This low redshift, combined with the apparent brightness $(B=16.0)$ of the BAL QSO PG $0946+301$, provides a unique opportunity for detailed observation of an object of this type. Such observations have previously been difficult or impossible due to the higher redshift and apparent faintness of BAL QSOs in general $(B>17)$.
UV and red optical spectroscopy to look for other absorption troughs, polarization measurements of the emission- and absorption-line profiles, and high-resolution studies of the absorption structure may be possible for this object. These observations would be invaluable not only for the study of PG $0946+301$ itself but also of BAL QSOs as a whole, since the object appears "typical" of the class in all but its redshift.

I gratefully acknowledge the UK Science and Engineering Research Council for financial support on a NATO fellowship. It is a great pleasure to extend my thanks to the director and staff of Steward Observatory for their hospitality during the tenure of my fellowship.

\section{REFERENCES}

Burbidge, E. M. 1970, Ap. J. (Letters), 155, L43.

Foltz, C. B., Wilkes, B. J., Weymann, R. J., and Turnshek, D. A. $1983 a$, Pub. A.S.P., 95, 341

1983b, Pub. A.S.P., 95, 603

Green, R. F. 1984, private communication

Lynds, C. R. 1967, Ap. J., 147, 396.

Oke, J. B. 1974, Ap. J. Suppl., 27, 21

Schmidt, G. D. 1984, private communication

Schmidt, M., and Green, R. F. 1983, Ap. J., 269, 352
Stockman, H. S., Angel, J. R. P., and Hier, R. G. 1981, Ap. J., 243, 404. Turnshek, D. A. 1984, Ap. J., 280, 51.

Weymann, R. J., Carswell, R. F., and Smith, M. G. 1981, Ann. Rev. Astr. Ap., 19, 41 .

Weymann, R. J., and Foltz, C. B. 1983, in Quasars and Gravitational Lenses (Proc. 24th Liège Int. Ap. Colloq.) (Liège: Université de Liège, Institut d'Astrophysique), p. 538.

Wilkes, B. J. 1984, M.N.R.A.S., 207, 73.

Note added in proof.-The discovery of another low-redshift BAL, PG $1700+518(z=0.292, b=15.43)$, has recently been reported by M. Pettini and A. Boksenberg (1984, Gemini, Vol. 11, July).

Belinda J. Wilkes: High Energy Astrophysics Division, Center for Astrophysics, 60 Garden Street, Cambridge, MA 02138 